\title{
Towards a Holistic View Of Security and Safety of Enterprise Information and Communication Technologies: Adapting to a Changing Paradigm
}

\author{
Dr. Klaus Brunnstein \\ Professor for Application of Informatics \\ University of Hamburg, Germany
}

Invited Paper for SEC97 (Copenhagen, May 16, 1997)

\begin{abstract}
When enterprises heavily rely upon proper working of Information and Communication Technologies (ICTs), they often experience shortcomings in programs and systems, failing availability and unreliable access through networks as major drawback in their operation, with possible effects on productivity and profitability. Traditional "security“ addresses some of these aspects but, based on its military model (Bell LaPadula), essential requirements are seriously missing.
\end{abstract}

With view towards distributed enterprise work, the paper analyses why basic concepts of "traditional security“ fail to meet these requirements. On this basis, it is postulated that holistic „sikerhet“ combining traditional security and safety is needed for ICT based enterprises. This concept requires improved professional education and awareness, but will also need more user and public awareness.

\section{Towards „Information Societies“: Dependability of IT-based Enterprises}

Roughly 50 years after James Watt's basic patent on steam-driven engines (1765), the development of industrial technologies had gained sufficient momentum to subsequently change economies, societies and states into what was later called „Industrial Societies“. With the deployment of „loco-motives“, economies overcame local boundaries to develop mass transportation and thus produce industrial chains (though not net-works) from raw material to advanced products and customers. The original „steam-driven“ engine was supplemented and later replaced by more advanced engines, based on liquid energy and electricity. Rather soon after their invention, as new enterprises and major branches of industrial economies developped, customers became significantly dependent upon the proper work of industrial products and methods.

Though industrial engines - driven by vapor, gasoline, or electricity - had their specific development cycles (Kondratieff's cycles of industrial development), one 
common characteristic was concerned with „quality" of related products. At least in their early phases, industrial products were rather "unreliable“ and often „dys-functional“. Only 100 years after their invention, when steam engines still tended exploding and producing significant damage, manufacturers developped some quality measures in design, production and maintenance of these engines to reduce risks for their users and environment. Only in those stages, quality measures were assessed and related organisations (dominated by the producers, rather than customers) developped to assess and assert ,product quality“. Almost 100 years after the invention of gasoline-driven engines $(\mathrm{N}$. Otto: 1867), cars were manufactured with inherent dys-functionalities. It was only when a customer attorney (Ralph Nader) publicly notified that such products were „unsafe at any speed", that quality of these industrial products was improved, enforced by developping „customer protection“ (subsequently implemented in national laws). In similar (thoug sometimes shorter) cycles, other engines (such as nuclear reactors) were experienced to be unreliable.

Almost 200 years after the advent of the ,industrial age“ (and roughly 20 years after its invention: e.g. Konrad Zuse, 1939), the fast proliferation of computers into enterprises lead some advanced industrial economies to new horizons of some "Information Era". It is assumed that the engine governing this development - ,the computer" - is an implementation of „human reasoning“. One application was to introduce new production methods (e.g. early versions of Computer-Integrated Manufacturing), thus enriching traditional industrial production methods in design, production, distribution and market access. In another direction, traditional information-related products such as monetarian services and insurances were adapted to computers for „informational usage“. Early adaptors (e.g. banks, insurances) were limited by initial (main-frame) computing technologies. With the advent of small, cheap and easy-to-handle „Personal Computers" (PCs) and with the development of computerized communication, new ,information products" and related production methods developped, which required and stimulated „Information Infrastructures“. IntraNets and InterNetworking is postulated to require and enforce a new paradigm of "distributed information work" which subsequently leads to new information engines (e.g. ,agent“ technologies) and more complex information products. These developments have even begun to change the world economic order into an ICT-supported „global economy“, and it has visible effects on vanishing abilities of national societies to enforce laws and maintain their values.

Only 60 years after the advent of „Computers“, and about 30 years after early developments of computerized enterprise communications (e.g. in inter-bank communication), many large to small enterprises have become dependent upon availability, reliability and proper work of computers and networks. Deficiencies in design, implementation, distribution and manufacturing of ICT- 
related products - esp. in their ,soft“ forms - lead to major losses in controllability, efficiency and values of information products and their ability to communicate. Many forms of ,risks“ materialize every day (see ACMs „Risk Forum"), and it is the ,,application side“, i.e. large enterprises and individual customers which have to pay.

As in related industrial phases, ICT quality in most areas is still underdevelopped. Even worse: ICT experts too often do not sufficiently care for quality! Though it is well known that contemporary PCs do NOT protect sensitive information and ICT processes, almost 100 millions are in daily enterprise use. Though it is well known that Internet is full of technical deficiencies (supporting hacking and massive scans, spoofing, sniffing and data hijacking, to name only few), ICT experts advise enterprises to communicate sensitive information via Internet and computerized telephone systems. New ICT products - e.g. script-based office systems - introduce and proliferate new risks (e.g. „macro viruses“, „malicious agents“ such as hostile JAVA applets and ActiveX controls) but almost no user cares. As in early industrial phases, „user requirements" are NOT defined by users but included in the specifications of ICT manufacturers. User unawareness materializes in ubiquitous software ,guarantee forms“ which every user must accept; such texts essentially say that users are themselves responsible for any malicious side-effect which the software produces, and that users at best get another copy of the deficitarian software which crashed their programs and data and destroyed results of hard work.

Many users tend to agree that it is high time to develop awareness among users about their dependency upon ICT, but users feel helpless. In a fatalistic approach quoting experiences of industrial evolutions, one could wait another 40 years until the problem is "sufficiently mature" (that means: damages have become sufficiently serious and ubiquitous) to enforce changes. In a different approach, this paper argues for a rational solution: it is the ICT profession (including related manufacturers, and lead by professional bodies such as IFIP) which must understand its shortcomings and which must improve ICT quality by developing new visions of how to support the application side in mastering their problems.

\section{2. „Security“: The Traditional Paradigm:}

When potential shortcomings of new ICT products (to name some recent examples: JAVA and ActiveX) are discussed, manufacturers (such as SUN and MicroSoft) tend to argue that they have designed and implemented, ,proper security". It is therefore worthwhile to understand security under the auspices of enterprises heading towards distributed information work. 
Essential concepts of „Security“ can be illustrated using the model of a military fortress (generally: a system requiring some protection of sensitive proxesses and objects) which is situated in an alien, foreign environment (some books on security illustrate their object with pictures such as the "Tower of London"). Those in the fortress are the „good guys" which have to protect themselves and their „goods“ against the „bad guys“ outside. Anybody from outside requiring access to the fortress is regarded suspicious. According to this model (which is mathematically well described by Bell and LaPadula in their famous BLPmodel), adequate protection against attackers (hackers, crackers) as well as surveyal (auditing) is essential. Consequently, access control (Identification and Authentication, I\&A) is a major issue, which regards any individual as potentially suspect and therefore requiring positive authentication (e.g. by analysing bio-metrical personal characteristica). Any possibly "covert" access through some hidden channel must be detected or, even better, excluded in the design of the fortress. Authenticity of outsiders must be visibly (e.g. by assigning „labels“) carried; to avoid risks of label manipulation, „label integrity“ must be assured.

Within the "protected environment", there are differences in „trustworthiness" of the good guys. Lower levels are trustworthy in fulfilling special operations but they are not equally trustworthy concerning information and decision-making. Senior insiders (e.g. high officers) carry sensitive information which require more rights to access information and make decisions though not always to perform operations. Consequently, „rights“ must be administered, and proper usage must be enforced (e.g. in design or by proper control) and surveyed (auditing). Strict rules forbid to set-up information channels where information may leak to less trustworthy insiders (in BLP-model: „no writedown") or even outsiders; on the other side, the flow of essential information must be guaranteed to reach only responsible insiders (BLP: „no read-up“).

The model of responsibility and required trust is structured like a pyramid: few persons on higher levels require higher trustworthiness as they have access to sensitive information and decide about sensitive actions. On the basis of this pyramid, many persons act on a comparably low level of required trust. One beneficial aspect of this pyramid structure is that it is easy to implement: control for higher risks (which requires higher efforts) may concentrate on few persons, whereas the mass of control processes can be performed on lower levels of requirements. This beneficial characteristic reduces the control overhead and guarantees, to some degree, a suitable performance/cost ratio.

In applying this model to ICT, „security“ was somewhat formally described in National Computer Security Center's „spectral series“, covering single system security („Trusted Computer Security Evaluation Criteria“, TCSEC alias Orange Book) and its „interpretations“ concerning interconnected single systems 
(„Trusted Network Interpretation“, TNI alias Red Book), databases („Trusted DataBase Management Systems Interpretation“, TDBI alias Lavender Book) and others. For different levels of „required trustworthiness“, systems can be evaluated and possibly certified to reach specific levels of "security“ (ranging from $\mathrm{C}$ to $\mathrm{B}$ to $\mathrm{A}$, with „beyond $\mathrm{Al}$ “ reserved for adaptations to unknown requirements). Based on these criteria (and requests of some US institutions for their usage), this model of security is implemented in some main-frame operating systems, on higher levels (B) such as MULTICS with its central „reference monitor“ controlling any access and action, or with less rigid mechanisms (C-level) such as IBMs MVS (with RACF). Compared to the mass of ICT products (esp. in office software areas), rather few systems and products have been evaluated according to TCSEC schemes, and even less apply TNI criteria. Recently, one client/server system used in many enterprises (Windows NT) has been quoted to be certified at $\mathrm{C} 2$, but only in its stand-alone facilities.

\section{Shortcomings of the Traditional „Security“ Paradigm:}

Even in the 1980s when distributed information processing was somewhat „behind the horizon", it was argued that the inherent military model was hardly applicable to enterprise computing. Indeed, concepts of enterprise work seems to be rather different from military concepts. One major diufference concerns access to and work with sensitive data: work on design data (e.g. of a product), personnel data (e.g. of customers or employees) and market data is accomplished by the lower-level employees, on the basis of the pyramid of responsibility. On the upper side of the responsibility pyramid, senior managers have less access to operationally sensitive information but use, e.g. in enterprise decision, highly condensed information. An enterprise may significantly depend upon both kinds of information - the daily operational and the decision-oriented ones. In traditional terms, users on lower levels must therefore be as intensely controlled as higher levels; this implies either significant mass-problems in control mechanisms (e.g. zillions of audit data, performance problems in I\&A) or reductions of control requirements. Usually, enterprises take the latter approach, e.g. in reducing authentication to low-level passwords, in switching audit trails off or in simply storing audit data without any evaluation.

Moreover, the assumption that insiders are good guys (if indeed applicable to the military) does not always hold for enterprises. Several case studies about computer crime have concluded that damage from inside attacks was significantly higher than from outside attacks (though this may change with developments of network-based ,information war" between enterprises which exploit weaknesses of networks). In traditional terms, outsiders can simply be hindered in accessing sensitive data by proper physical, logical and organisation means. On the other side, insiders - esp. when operating on low levels of 
„security“ - have many opportunities to access, manipulate or else misuse sensitive enterprise information. Moreover, unplanned damages e.g. by import and usage of malicious programs (,computer viruses“, „trojan horses“) and documents (,macro viruses") are regarded as higher risks for enterprise computing than hacker attacks.

In an attempt to model enterprise, security" requirements in a form comparable to the military fortress model, ,integrity“ of processes and data was postulated. Related models have been developped, e.g. by Biba and Lipner which tried (though with differing assumptions) to build upon the traditional BLPmodel. In another approach, the Clark-Wilson model attempted to describe the logical inter-dependency of enterprise transactions to develop some possibly formally provable model of integrity. Recently, Abrams and LaPadula developped a frame model which allows to import different - possibly incoherent - models into a ,general framework“.

Following such suggestions, traditional security criteria have been somewhat enhanced. In one direction, four national European security government offices developped the "Information Technology Security Evaluation Criteria“ (ITSEC). An essential merit of these criteria was to include integrity (though not clearly defined). Moreover, several aspects of communication (e.g. integrity and confidentiality) were added as new functions. Despite of the value of these further dimensions, IT-dependent enterprises have so far not experienced improved product quality in ITSEC directions. Indeed, one other innovation of ITSEC seems to produce major drawbacks in product quality. In TCSEC, specific security functions and the related assurance are closely bundled. ITSEC takes a different approach in separating functions (F) from „quality“ (E). In ITSEC, each system or product can be described by a pair $(F, E)$; it has not been properly analysed whether functional requirements and quality of products are sufficiently independent (mathematically: orthogonal) to serve as essential descriptions.

One beneficial effect of „unbundling“ security funtionality from quality of implementation could be that new functions (e.g. an AntiVirus functionality: FAV) can easily be added. In reality, few such additions have been published over the last years. Moreover, breaking quality from functionality has now a dubious side-effect: products can receive an ITSEC certificate without any explicit functionality. So far, several products have certificates such as „medium quality“ which classifies a producers attitude and ability rather than making its products comparable to others. In several cases, US-based products have been certified which very probably would NEVER be certifiable according to Orange book.

Presently, another attempt - this time jointly by North-American and European state organisations - aims at developping „Common Criteria“. Fresh ideas such 
as ,protection profiles“ and a much larger set of functionality classes wait for being applied to relevant products. Though it will be interesting to observe whether this joint activity can lead to ICT products of higher quality, one native problem of all those criteria quoted (TCSEC, ITSEC, CC) is that they have essentially been developped by government authorities with long experience and strong interest in military models; experiences in enterprise computing and networking have rather rarely been represented in related commissions. This diminishes the likelihood that new approaches help understanding enterprise dependability problems.

All security models so far are essentially based on the assumption that ICT work is performed on a set of single installations whose characteristics and interdependencies can be informally or formally described. Clearly, this assumption is no longer valid when some dynamically adapting network replaces „the computers“. To give one example: when some „agent" such as an "information broker" searches an Intranet for specifical information, it is neither possible nor feasible to know the actual network structure nor to control any information access on foreign systems; indeed, most users of such agents will never know whether this agent uses plug-ins in other systems to read local information. The complexity of the network and its associated production methods excludes "proper control“, though the „quality“ of the derived information depends strongly upon the quality of those production methods.

It should not surprise that classical security concepts (including certified products) are not highly relevant (if at all) in enterprise information work. Indeed, an ITSEC certified product seems to have no advantage over non-certified products in enterprises in general. One reason may be that manufacturers tend to declare their products as (e.g. $\mathrm{C} 1$ or F-C1/E1) secure though they are at best "designed at" that level. One other reason may be that certification strongly relies upon information presented from manufacturers; on such basis, one can hardly be surprised that certified products fail even in certified areas (one "nice“ example was an AntiVirus product which, after having been certified by German Information Security Agency, was proven to distribute rather than detect viruses which it ,improperly" handled; although this was made public, the certificate was NOT withdrawn).

Following the methodological inadequacies described, the state of "security“ in many enterprises is also "inadequate“. Many enterprises rely on software products which are - if working with some stability - D-level (TCSEC: „no security“). This applies esp. to Intel/Microsoft based „DOS/Windows“ systems (with Windows NT fulfilling, in principle, C-functionalities, in local use) and Macintosh-OS, but also to most UNIX systems (IBMs AIX and HewlettPackards HP-UX are certified at C-level, but their network components are so 
buggy that Computer Emergency Response Teams have to distribute „warnings“ almost weekly). Generally, practice in I\&A is as deficient as auditing.

Moreover, the situation in networks is even worse. Neither TNI nor communication-oriented functionalities in ITSEC have brought major improvements in security. Whereas SWIFT as inter-banking network had some built-in security mechanisms, Internet banking is based on a network with essentially NO BUILT-IN security. Indeed, original concepts of ARPANET (though financed and stimulated by military organisations) are „insecure at any level“" (to adapt Ralph Nader's decription of some industrial products); the only relevant design goal was that partial networks survive failures at other locations. When new information products such as Internet Commerce and Internet Banking are put on such insecure technologies, either additional means are needed or additional risks will unavoidably materialize.

Some security experts argue that good encryption can overcome risks in otherwise insecure networks. This approach assumes that encryption mechanisms can be made unbreakable (which is in principle achievable as such methods as RSA ciphers with sufficient keylength are practically unbreakable for some time); moreover, this approach would be successful IF crypto-key management can be implemented with sufficient reliability and trustworthiness (which is harder than just implementing a good algorithm), AND IF keys can be protected against attempts of governments to force manufacturers implementing methods that allow states to decipher encrypted messages. Discussions about "secure encryption“ of the European mobile telephone communication (A3/A8 algorithms being said to be unbreakable but now allowing deciphering) and successful requirements of European governments to break encrypted communication show that users canNOT rely upon proper encryption. Actual discussions about crypto-laws in USA and Europe point in the same direction.

Conclusion: neither the dominant understanding of „security “ nor its enforcement by criteria is adequate for IT-based enterprises.

\section{Safety and Security: Adequate Paradigm for ICT-dependent Enterprises}

Essential requirements of enterprises come from areas which have been overseen in security discussions. Apart from the inadequate dominance of military "security", one reason is the dominant Anglo-Saxon language which distinguishes between ,security“ and "safety“. In other European languages, such as Scandinavian (Denish, Norvegian, Swedish) and German, the central term (,sikerhet", „Sicherheit") includes BOTH Security AND Safety which I subsequently call „Sikerhet“. 
Generalized Sikerhet requirements include the following "dimensions“ applied to „ICT objects“ such as systems, programs, functions, services, data, structures, interdependencies etc:

Functionality: an ICT object perfoms its function always ,as required“,

Consistency: an ICT object is always consistent with its specification or definition,

Persistency: an ICT object is changed during its life-time only by explicitly permitted action,

Reliability: all functions performed on an ICT object are always equally performed under equal constraints,

Availability: all ICT objects are available when needed, and

Confidentiality: no illegal access to or use of an ICT object is possible.

Some of these aspects relate to „traditional security“, such as confidentiality and integrity (which is generalized to persistency). Others have played major roles in other areas, such as control of industrial processes or application in high-risk areas such as medical ICT, control of infrastructures such as nuclear energy production, or control of life-sensitive transportation systems such a automatic vehicle control or Flight Management Guidance Systems (alias EFCS = Electronic Flight Control Systems). Related methodological requirements have been called „Safety“ or „Dependability“ requirements. Indeed, all these aspects - rather than the subset of "traditional security“ - are relevant for ICT-based work. Therefore, a holistic view is needed (including a holistic name).

When one compares the methods applied in "traditional safety" (e.g. as used in developments of industrial application) with those in "traditional security", one finds that formal methods are applied in both areas, but with different sets of assumptions - and consequently with different success. The different approach can be condensed to the following antagonistic positions:

Where users are seriously aware of their mission and criticality (as in many applications of safety), users tend to prescribe what they require. Formal methods are applied in modern applications to describe such requirements, which may also be used to assess whether actual products fulfil given requirements. 
Where manufacturers are more determined to bring their ideas and products to markets of many users, they tend to specify what users may find worthwhile to work with. Such specifications are mainly for internal purposes, and they are rarely formally given or certified.

In a holistic approach, one must not only combine dimensions to yield a problem/solution space of higher order, but one must show that the holistic picture may seriously win from combining methods from the sub-spaces. Indeed, one basic advantage of holistic "Sikerhet" is that both views may well be combined to a sequential process, to the advantage of ICT quality:

Step 1: Users of Sikerhet-related products describe their requirements.

Step 2: Manufacturers of Sikerhet-related products specify what their products offer and what they can guarantee.

Step 3: On the market of Sikerhets-related IT products, users compare their requirements with manufacturer specifications. When they match (at least partially), the manufacturer ,guarantees" the proper work of his product ,as specified“ within agreed limits.

This mechanism is somewhat comparable to methods of industrial quality assurance. It supports improved product certification such as developing „sikerhets criteria“" which may eventually be further developped from traditional security (e.g. by properly enhancing confidentiality from single and conneced to distributed systems) and traditional safety (e.g. prescriptions of certifications of industrial products, air transport systems or nuclear power stations). Moreover, orientation towards production of „sikerhets products“ based on ISO 9000 are also possible; some of these aspects (e.g. life-cycle orientation) are yet covered in ITSEC and Common Criteria.

\section{Outlook: Implementation of Holistic „Sikerhet":}

In order to implement Holistic „Sikerhet“, several processes must be started:

User awareness must develop to understand deficiencies of contemporary ICT. In some sense, this is an automatic process. With present InSecurity and UnSafety, it is just a matter of time that risks materialize to such a degree that customer protection becomes a matter of survival. The sad lesson (see 1) of industrial developments is that this may even happen at the expense of human lifes. One would hope that such serious con-sequences may be avoided in a technology which is based on human ratio. 
Professional education will play an important role in planning, implementing and maintaining ICT on higher levels of "Sikerhet". Methods such as specification techniques and tools are available but professional education is still on the level of medium level programming as languages such as $\mathrm{C} / \mathrm{C}++$ and Visual Basic are major carriers of innovation.

In Security, only few universities offer coherent courses on Security and Safety; in Germany, Hamburg University's related four-semester course (presently finishing its 4th cycle, with participation of over 60 students) is the only one in an Informatics faculty. Few other European universities (in Sweden, UK, Autria and Greece) are also offering related courses; in a joint European project (ERASMUS), several universities exchange both teachers and students to develop professional education. Within IFIP, a TC-11 working group also works on harmonising curricula.

Professional awareness must also be developped for experts which areactive in ICT industry to improve their previous - unsufficient education.Apart from updating the professional knowledge at related conferences, national Computer Societies should play a major role.

Public awareness will probably only develop when the public realizes how insufficient contemporary ICT is to reach the publicly propagated goals of "Information Societies“. Presently, politicians in USA and Europe assume that basic technologies such as PCs, multimedia and Internet work,as assumed". Only when writers and politicians experience themselves how unreliable and unavailable Internet connections often are, that data from Internet are often wrong and search engines produce misleading results, they will learn that their favorite technologies need „shaping“. This will include legal regulations to enforce customer protection and reduce effects of failing ICT products.

In historical perspectives (which disregards individual and enterprise life stories), concern about and requests for „sikerhet" will unavoidable grow. Every new report about failing programs (e.g. when some network carrier becomes inoperable for hours or days) or forgotten characteristics (such as dropped century figures in dates) will add to the public insight that present ICT does not perform what the public thinks. This will also diminish the respect for IT professionals. Instead of observing how the information engines „explode“, anticipatory action is the better advice. 


\section{References:}

This paper re-evaluates concepts of traditional security which have been published in well-known literature, broadly available in SEC conferences. The paper is based on the authors book on "Information Technology: Inherent Paradigms and Risks for Future Information Societies“ (in preparation). 